\title{
Primary pancreatic small cell carcinoma diagnosed by endoscopic ultrasound-guided fine needle biopsy
}

\author{
Seung Yong Park ${ }^{1}$, Keum Ha Choi ${ }^{2}$, and Hyung Ku Chon ${ }^{3}$
}

${ }^{1}$ Department of Internal Medicine, Chonbuk National University Medical School, Jeonju; Departments of ${ }^{2}$ Pathology and ${ }^{3}$ Internal Medicine, Wonkwang University Hospital, Iksan, Korea

Received: July 13, 2018

Revised : August 14, 2018

Accepted: August 20, 2018

\section{Correspondence to} Hyung Ku Chon, M.D.

Tel: $+82-63-859-2564$

Fax: +82-63-855-2025

E-mail: gipb2592@wku.ac.kr
A 75-year-old man without underlying disease was referred to our department presenting with abdominal distension. Laboratory tests were unremarkable except for a significant elevation of carbohydrate antigen 19-9. An abdominal computed tomography (CT) scan demonstrated an approximately 8 $\mathrm{cm}$-sized mass with a necrotic portion in the pancreatic tail and multiple hypodense masses involving liver, spleen, and adrenal gland with massive ascites (Fig. 1 A and 1 B). A chest CT scan showed no evidence of pulmonary tumor. A positron emission tomography scan revealed intense hypermetabolic uptake in the pancreatic tail, liver, spleen, both adrenal glands, and peritoneum (Fig. ${ }_{1 C)}$. Endoscopic ultrasonography (EUS) showed an ill-defined heterogeneous mass in the pancreatic tail with peripancreatic and perihilar lymph node enlargement. EUS-guided fine needle biopsy (FNB) was performed with $22-\mathrm{G}$ core needle on the pancreatic mass. On histology, small round cells with nuclear molding and crush artifacts (Fig. 2A). Immunohistochemical (IHC) staining was negative for thyroid transcription factor (TTF-1), leucocyte common antigen, and positive for $\mathrm{CD}_{5} 6$, which was compatible with primary pancreatic small cell carcinoma (PPSCC) (Fig. 2B and $2 \mathrm{C})$. The patient refused palliative
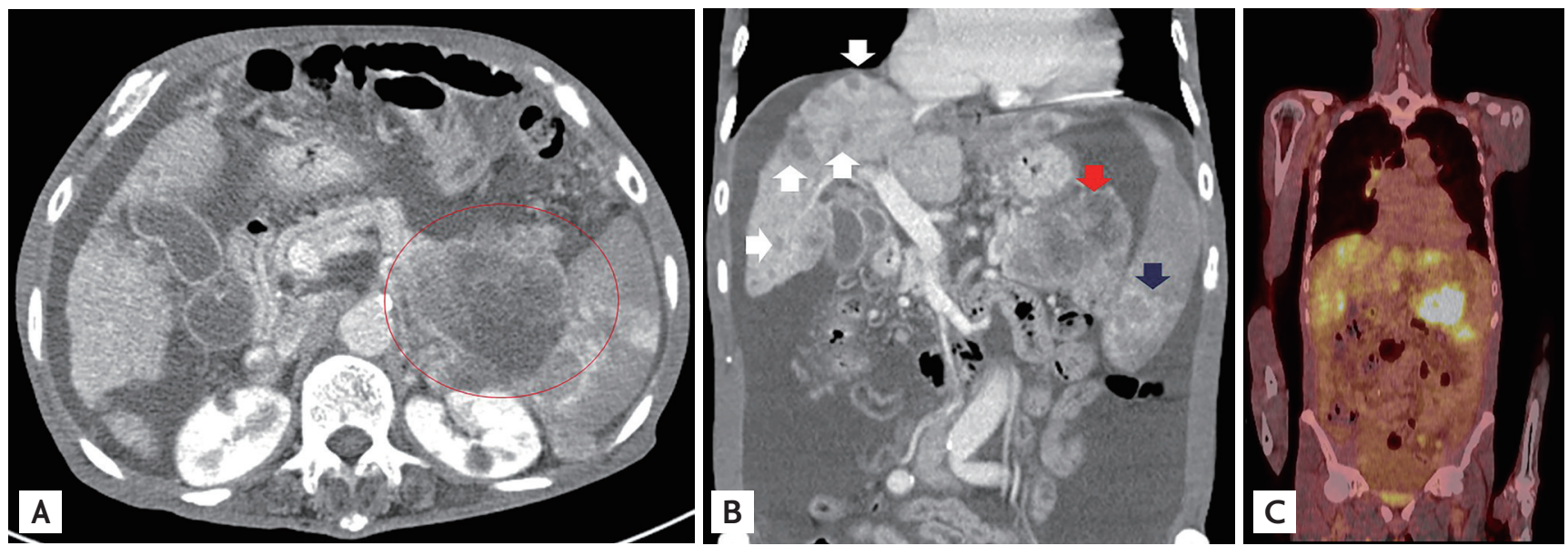

Figure 1. (A) Computed tomography of the abdomen showed a large pancreatic mass involving spleen and adrenal glands (red circle) with massive ascites. (B) On coronal view, large pancreatic mass (red arrow), splenic mass (blue arrow), and multiple hypo-dense masses (white arrows) in the liver were seen. (C) Positron emission tomography scan demonstrating $18 \mathrm{~F}-$-fluorodeoxyglucose activity in the pancreatic tail, liver, spleen, and multiple lymph nodes. 

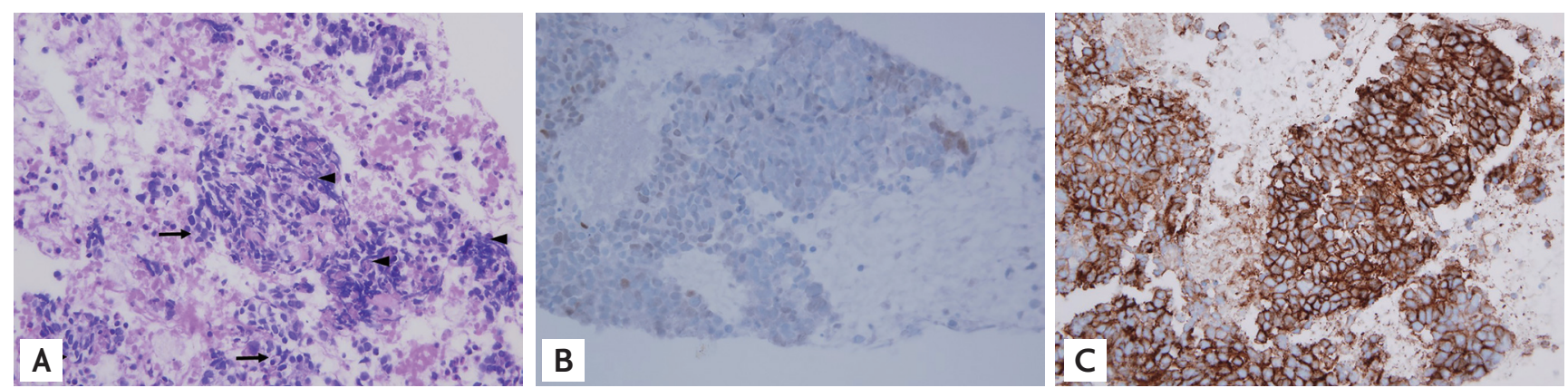

Figure 2. (A) Small round cells show nuclear molding (arrows) and crush artifacts (arrowheads) (H\&E, $\times 400)$. (B) Immunohistochemical staining reveals tumor cells negative for thyroid transcription factor 1 (TTF-1, $\times 400$ ) and (C) positive for CD 56 (CD56, $\times 400)$.

chemotherapy and expired 3 weeks later.

PPSCC is extremely rare, accounting for less than $1 \%$ of primary pancreatic malignancies. PPSCC has aggressive behavior and is usually diagnosed at an advanced stage. Therefore, the survival duration may be very short, although there are several reports of patients with PPSCC achieving partial or complete remission after combination chemotherapy. Cross-sectional imaging and IHC staining by EUS-FNB of obtained tissues are useful for determining whether a pancreatic small cell carcinoma is primary or metastatic. PPSCC diagnosis can be made in the absence of an abnormal pulmonary pathologic condition and is compatible with histopathologic results showing round to spindle-shaped small cells immunoreactive for $\mathrm{CD}_{5} 6$, chromogranin, and synaptophysin, but negative for TTF-1. Here, we report a rare case of PPSCC with poor prognosis and diagnosed by EUS-FNB using a 22-G core needle.

Written informed consent were obtained.

\section{Conflict of interest}

No potential conflict of interest relevant to this article was reported.

\section{Acknowledgments}

This report was supported by Wonkwang University in 2019. 